\title{
Photoexcitation in thin films deposited on silicon substrates by reactive pulsed laser ablation
}

\author{
A. Giardini Guidoni, ${ }^{1,2}$ V. Marotta, ${ }^{1}$ S. Orlando, ${ }^{1, \dagger}$ and G. P. Parisi ${ }^{1}$ \\ ${ }^{1}$ CNR - Istituto per i Materiali Speciali, Zona Industriale, I-85050 Tito Scalo (PZ), Italy \\ 2 Dipartimento di Chimica, Universita' di Roma "La Sapienza", P.le A. Moro 5, I-00185 Roma, Italy
}

\begin{abstract}
Reactive pulsed laser ablation is a very interesting method to deposit thin films of several materials and compounds such as oxides, nitrides, semiconductors and superconductors. This technique relies on photoablation of pure elements, or a mixture of materials, with simultaneous exposure to a reactive atmosphere. In the case of oxides, reactions between the laser vaporized metals and oxygen lead to the formation of intermediate complexes and finally to oxide thin films. The reactivity of the plume has been already studied by our group in other oxides and nitrides productions and ascertained by Time of Flight Mass Spectrometry measurements [1].

Thin films of semiconducting oxides such as $\mathrm{In}_{2} \mathrm{O}_{3}, \mathrm{SnO}_{2}$, and multilayers of these two compounds have been deposited by Reactive Pulsed Laser Ablation, with the aim to evaluate the behaviour of such films under variable halogen lamp illumination.

Deposition of these thin films has been carried out by a frequency doubled Nd-YAG laser (wavelength = $532 \mathrm{~nm}$ ) on Silicon (100) substrates. A comparison, among indium oxide, tin oxide, and multilayers of indium and tin oxides, has been performed. The influence of physical parameters, such as substrate temperature and oxygen pressure in the deposition chamber, has been investigated. The deposited films have been characterized by Electric Resistance measurements.
\end{abstract}

\section{INTRODUCTION}

Studies on semiconductor oxides are growing very fast because of successful production of these ceramic materials as detecting devices. Metal oxide coatings are widely used as transparent resistive heaters, electrical electrodes for flat panel display and electrochromic devices, antireflecting and high refraction index materials for optical applications, and gas sensors. These thin films are usually produced by Chemical Vapor Deposition [2], Microwave discharge, Molecular Beam Epitaxy [3, 4], sputtering [5] and sol-gel process [6]. Another attractive choice for the preparation of these films is the reactive pulsed laser ablation and deposition (RPLAD). Pulsed laser deposition has been applied successfully in the past to deposit many materials such as semi- and super-conductors, nitrides and oxides [7]. This technique relies on photoablation of pure elements, or a mixture of materials, with simultaneous exposure to a reactive atmosphere. In the case of oxides, reactions between the laser vaporized metals and oxygen lead to the formation of intermediate complexes $[1,8]$ and finally to oxide thin films. The RPLAD deposition of indium oxide and tin oxide thin films, and multilayers of these two compounds, has been performed with the aim to evaluate the behaviour of such films under variable halogen lamp illumination. The deposited films have been characterized by Electric Resistance measurements to understand the influence of physical parameters such as

${ }^{\dagger}$ E-mail: orlando@ims.pz.cnr.it substrate temperature and oxygen pressure.

\section{EXPERIMENTAL}

The laser ablation experiments were carried out in a multiport stainless steel vacuum chamber equipped with a gas inlet, a rotating multi-target and a heatable substrate holder. The deposition temperature can be varied from room temperature up to $700^{\circ} \mathrm{C}$. The fluence of a Quantel Nd:YAG 581 laser (wavelength = $532 \mathrm{~nm}$, pulse duration $=7 \mathrm{~ns}$, repetition rate $=$ $10 \mathrm{~Hz}$ ) has been kept at $8 \mathrm{~J} / \mathrm{cm}^{2}$. The laser impinges on the target, at $45^{\circ}$ with respect to the normal, in a static atmosphere of $\mathrm{O}_{2}$ at different pressures.

Thin films of indium oxide and tin oxide, and twolayered films ( $\mathrm{Sn}$ - In) have been deposited on Silicon substrates (100) by reaction of laser ablated metallic targets of indium and tin in presence of oxygen, at different substrate temperatures [9]. The bi-layered " $5 \mathrm{Sn}$ - 5In" type has been obtained depositing the first layer of tin oxide for 5 minutes, corresponding to a thickness of about 0.4 micron, and then for other 5 minutes the indium oxide layer (thickness 0.5 micron). Analogously the "10Sn - 5In" type has been deposited.

$\mathrm{X}$ ray spectra were detected by a Siemens D5000 diffractometer using the $K \alpha$ line of a $\mathrm{Cu}$ target as an $X$ ray source. A four-contact probe, based on a Keithley 224 Programmable Current Source and a Keithley 182 Sensitive Digital Voltmeter, has been utilized to evaluate the resistance variation of thin films when illuminated by a halogen variable lamp, up to 11000 lux. The lumi- 
nous flux density of the halogen lamp has been roughly measured by an ISO-TECH ILM 350 Digital Light Meter with sensitivity centered at $550 \mathrm{~nm}$.

\section{RESULTS AND DISCUSSION}

For our group, the first aim to deposit thin films of indium oxide, tin oxide and multilayers of these two compounds was to demonstrate the validity of RPLAD in the production of such thin films as toxic gas sensors [911]. The multilayered deposition has been utilized to enhance the detection capability of the films.

Performing the electric characterization of these thin films, to evaluate their response when got in contact with the toxic gas, we noted a very fast variation of resistivity due to the illuminance condition during the measurements. So we had to carry out all the electric measurements in the dark, as deep as possible. Starting from this experimental evidence, we have tried to evaluate just the variation of resistance of our films when illuminated by a halogen lamp, starting from $100 \mathrm{~lx}$ till about $11 \mathrm{Klx}$, each step lasting about 50 seconds. In this paper we report the very preliminary results obtained. We do not perform any dynamical measurement on the quickness of the response, due to the present inadequacy of our measuring apparatus.

In order to compare the results from different thin films, we have calculated a normalized resistance, i.e. the ratio between the measured resistance and a reference value, keeping as reference of each sample its resistance measured at $100 \mathrm{~lx}$ of illuminance:

$$
R_{\text {ref. }}^{\mathrm{s}}=R^{\mathrm{s}}(100 \mathrm{~lx}) \text {. }
$$

Just as example, in Figure 1 we report the resistance behaviour of an indium oxide thin film, in the range 20$11000 \mathrm{~lx}$. All the other presented figures (Figures 2-4)

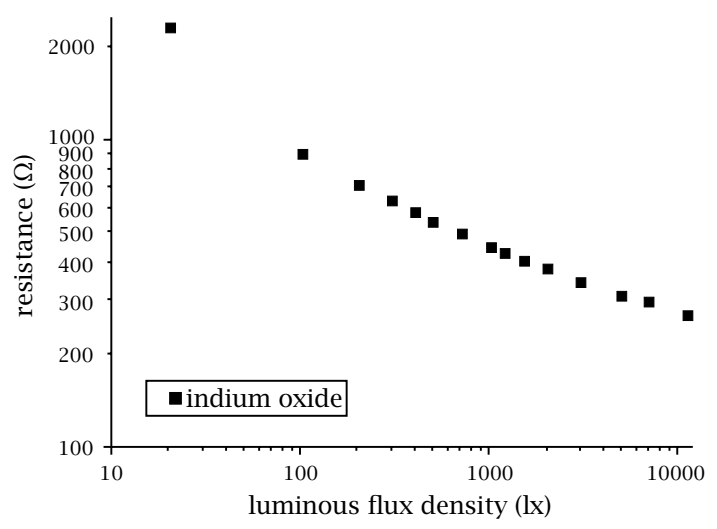

Figure 1. Resistance behaviour of indium oxide thin film when illuminated by a halogen lamp. Deposition parameters: fluence $8 \mathrm{~J} / \mathrm{cm}^{2}$, substrate temperature $500^{\circ} \mathrm{C}$, oxygen pressure $100 \mathrm{~Pa}$. report the normalized resistance.

The importance of the Si (100) substrate was clear just from the beginning. In fact, we attempted to measure similar effect in indium oxide films deposited on $\mathrm{MgO}$ (100) substrate but there was no evidence of light influence, except for the resistance variation due to the unavoidable heating of the samples by the lamp.

The heating of the thin films is always present in all the spectra recorded. Its presence is shown by the, more or less evident, depletion of the normalized resistance above some Klx of illuminance.

But the fast variation of the resistance due to the illumination by the halogen lamp is not only exclusively connected with the peculiar characteristics of silicon. In fact, we found quite different responses when depositing different metal oxides or other compouds on Si (100) substrates.

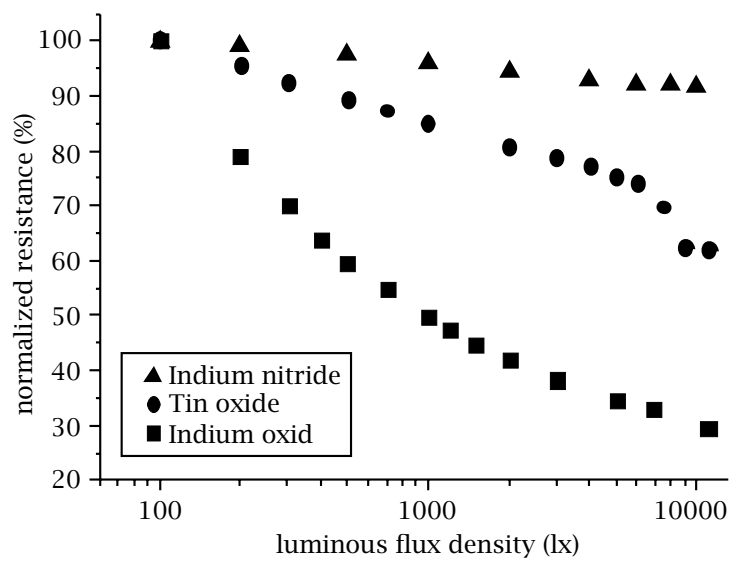

Figure 2. Normalized resistance of indium oxide, tin oxide and indium nitride. The indium nitride is quite insesitive with respect to metal oxides. Deposition parameters: fluence $8 \mathrm{~J} / \mathrm{cm}^{2}$, substrate temperature $500^{\circ} \mathrm{C}$, gas pressure $200 \mathrm{~Pa}$.

In Figure 2 it is reported the normalized resistance of three single layer samples: indium oxide, tin oxide and indium nitride. The indium nitride is shown just to evidence its negligeable response with respect to metal oxides, and to demonstrate that the behaviour of such films do not depend only by the Si (100) substrate. This evidence let us suppose that the interphase silicon-oxide should be probably the very important site where the impinging light is absorbed. Between the two oxides investigated, indium oxide show the much more evident response to the halogen lamp illumination.

A preliminary screening was performed to evaluate the influence of oxygen pressure during the depositions, ranging from 100 to $1000 \mathrm{~Pa}$. The results obtained from the bi-layered " $5 \mathrm{Sn}-5 \mathrm{In}$ " tin oxide-indium oxide multilayers are reported in Figure 3, as function of the oxygen pressure in the deposition chamber. The trend of the behaviour is quite evident, showing 


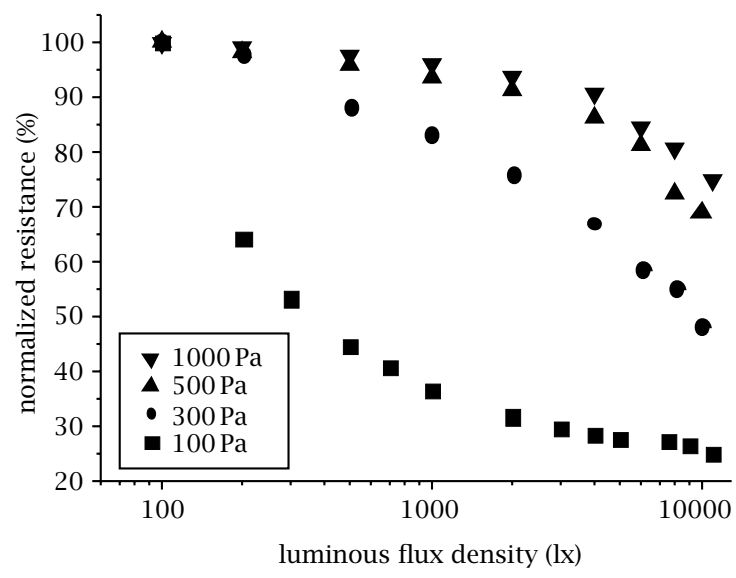

Figure 3. Normalized resistance of tin oxide-indium oxide "Sn5 - In5" multilayers as function of oxygen pressure in the deposition chamber. Deposition parameters: fluence $8 \mathrm{~J} / \mathrm{cm}^{2}$, substrate temperature $500^{\circ} \mathrm{C}$.

the best results at low oxygen pressure. The measurement of a sample deposited at 50 Pa showed a worst behaviour, probably due to a poor oxidation of the metal components in the gas phase. All films have been deposited at $8 \mathrm{~J} / \mathrm{cm}^{2}$ laser fluence and at $500{ }^{\circ} \mathrm{C}$ substrate temperature.

The trend shown in Figure 3, suggests that the energy of the particles ejected from the metallic target, travelling through a more rarefied buffer gas, could reach the substrate at a higher velocity and "damage" the surface of the silicon substrate creating a thin interphase layer much more sensitive to the light energy.

X-Ray Diffraction (XRD) analysis showed polycrystalline structures either in indium oxide and tin oxide single layer depositions and in bi-layered films [9, 10]. XRD patterns of " $5 \mathrm{Sn}-5 \mathrm{In}$ " thin film, produced at $1000 \mathrm{~Pa}$ oxygen pressure and $500{ }^{\circ} \mathrm{C}$ substrate temperature, showed several $\mathrm{SnO}_{2}$ (tetragonal) and $\mathrm{In}_{2} \mathrm{O}_{3}$ (cubic) diffraction peaks [11]. In this case, the tin oxide layer grows easily on silicon substrate at $1000 \mathrm{~Pa}$ oxygen pressure with respect to $200 \mathrm{~Pa}$ [11]. These facts could confirm the importance of the interphase layer to enhance the response at the impinging light.

In Figure 4 is reported the normalized resistance of tin oxide-indium oxide "Sn10 - In5" multilayers, deposited at different substrate temperature; the behaviour of such samples does not show a clear evolution. It seems that up to few Klx ( $<4 \mathrm{Klx})$ the bi-layered thin films deposited at $600{ }^{\circ} \mathrm{C}$ should have the best response to the light. At higher illuminance (> $4 \mathrm{Klx}$ ) the multilayers deposited at $500{ }^{\circ} \mathrm{C}$ show better results, even if the variation is not so evident. Probably, the interphase layers, of the samples deposited at substrate temperature in the range $400-600{ }^{\circ} \mathrm{C}$, do not differ very much among them.

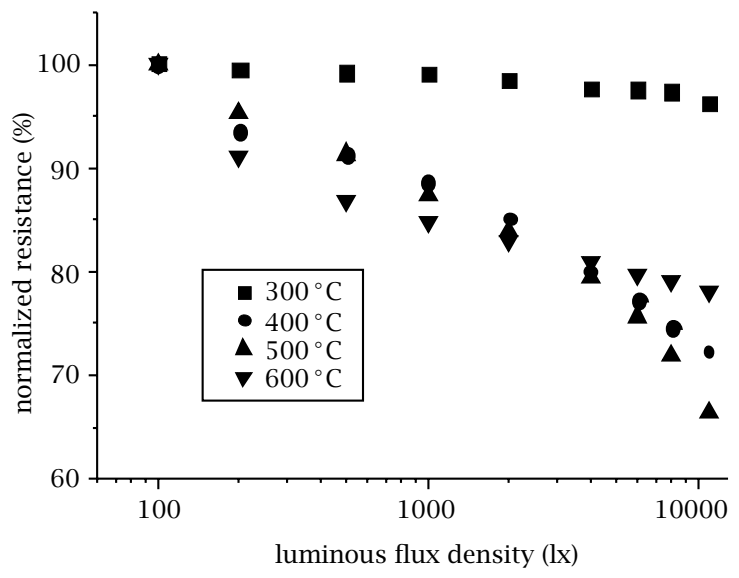

Figure 4. Normalized resistance of tin oxide-indium oxide "Sn10 - In5" multilayers as function of substrate temperature during the deposition. Deposition parameters: fluence $8 \mathrm{~J} / \mathrm{cm}^{2}$, oxygen pressure $200 \mathrm{~Pa}$.

\section{CONCLUSIONS}

In this paper we report preliminary results about the resistance variation of thin films (indium oxide, tin oxide, and multilayers Sn - In), grown on Si (100) substrates by reactive pulsed laser ablation and deposition, when illuminated by a halogen lamp. We noted a very fast variation of resistivity due to the illuminance condition during the measurements, performed by a four contact probe.

The results showed a straight dependence of the response to the illumination of oxide thin films deposited on Si (100) substrates. The silicon substrate is essential to show the fast behaviour under illumination but the enhancement of such effect depends on the thin film deposited on it. The single layer of indium oxide works better than tin oxide.

We have shown that multilayers tin-oxide indium oxide, deposited at $100 \mathrm{~Pa}$ oxygen pressure, have a more significant resistance variation, when they are exposed to a halogen lamp up to $12 \mathrm{Klx}$, with respect to the samples deposited at higher pressure.

The resistance variation of samples, deposited at different substrate temperatures, does not seem to be so dramatically dependent on this parameter.

Work is in progress to better understand the influence of other deposition parameter, such as laser fluence and wavelength, and to improve the measuring apparatus.

\section{ACKNOWLEDGEMENTS}

This work was partially supported by the italian CNRPF_MSTA2. The authors wish to thank Dr. Antonio Morone for his useful collaboration and discussion. 


\section{References}

[1] R. Teghil, V. Marotta, A. Giardini Guidoni, T. M. Di Palma, and C. Flamini, Appl. Surf. Sci. 138 (1999), 522.

[2] E. N. Eremin, L. I. Nekrasov, E. A. Rubtsova, M. V. Belova, V. L. Ivanter, L. N. Zacharov, and L. N. Petukhov, Russ. J. Phys. Chem. 56 (1982), 788.

[3] M. A. Khan, J. N. Kuznia, J. M. van Hove, D. T. Olsen, S. Krishnanku tty, and R. M. Kolbas, Appl. Phys. Lett. 58 (1991), 526.

[4] S. Nakamura, Y. Harada, and M. Seno, Appl. Phys. Lett. 58 (1991), 2021.

[5] G. Sberveglieri, P. Benussi, G. Coccoli, S. Groppelli, and P. Nelli, Thin Solid Films 186 (1990), 349.
[6] R. Rella, P. Siciliano, L. Vasanelli, C. Gerardi, and A. Licciulli, J. Appl. Phys. 83 (1998), 2369.

[7] D. B. Chrisey and G. K. Hubler (eds.), Pulsed Laser Deposition of Thin Films, John Wiley \& Sons, Inc., New York, 1994.

[8] A. Mele, D. Consalvo, D. Stranges, A. Giardini Guidoni, and R. Teghil, Int. J. Mass Spectrom. Ion Process 95 (1990), 359.

[9] V. Marotta, S. Orlando, G. P. Parisi, and A. Giardini, Appl. Surf. Sci. 154 (2000), 640.

[10] V. Marotta, S. Orlando, G. P. Parisi, and A. Giardini, Appl. Phys. A 69 [Suppl.] (1999), S675.

[11] V. Marotta, S. Orlando, G. P. Parisi, A. Giardini, G. Perna, A. M. Santoro, and V. Capozzi, Appl. Surf. Sci. 168 (2000), 141. 


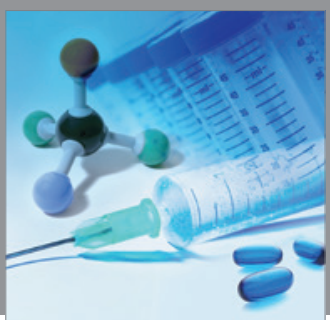

International Journal of

Medicinal Chemistry

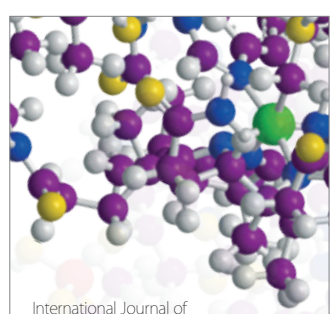

Carbohydrate Chemistry

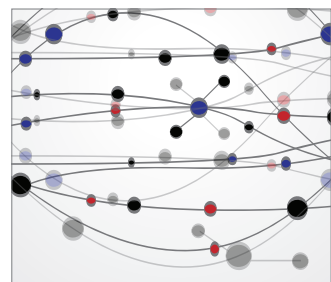

The Scientific World Journal
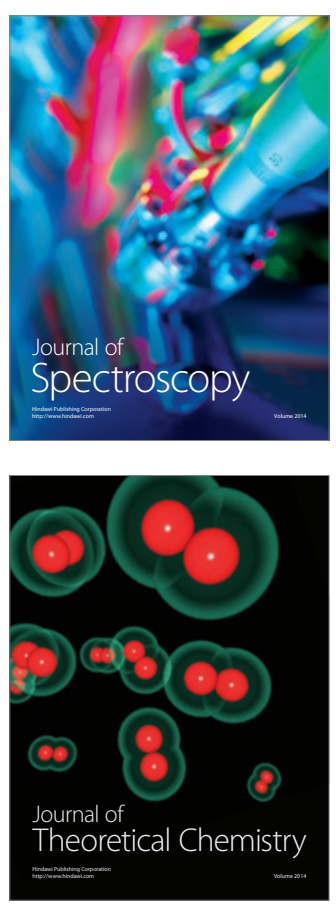
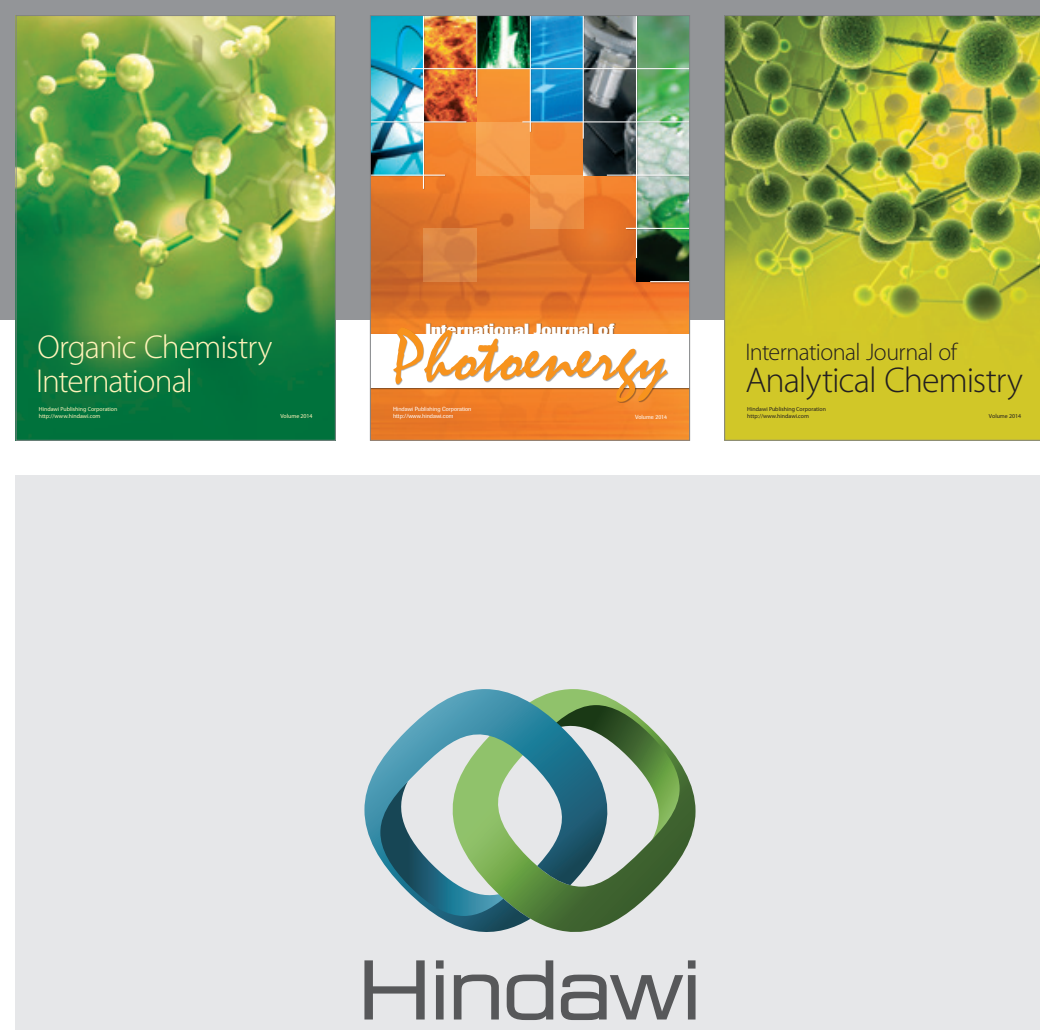

Submit your manuscripts at

http://www.hindawi.com
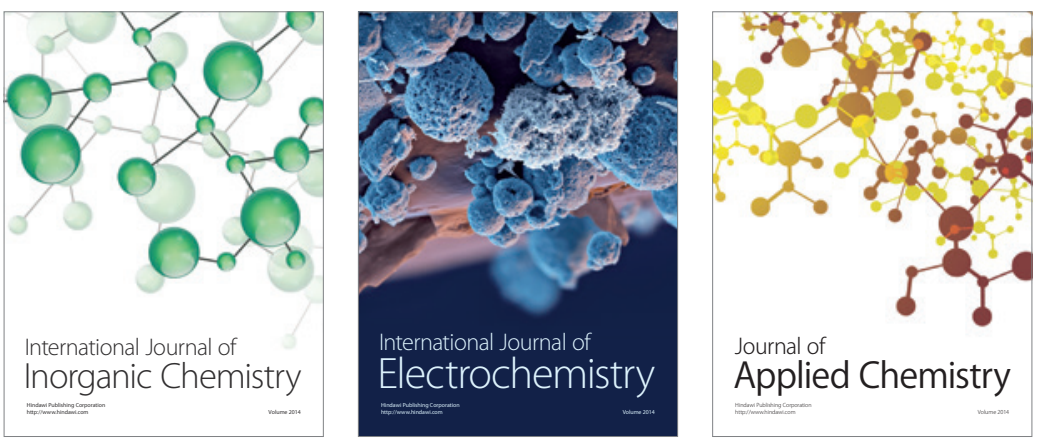

Journal of

Applied Chemistry
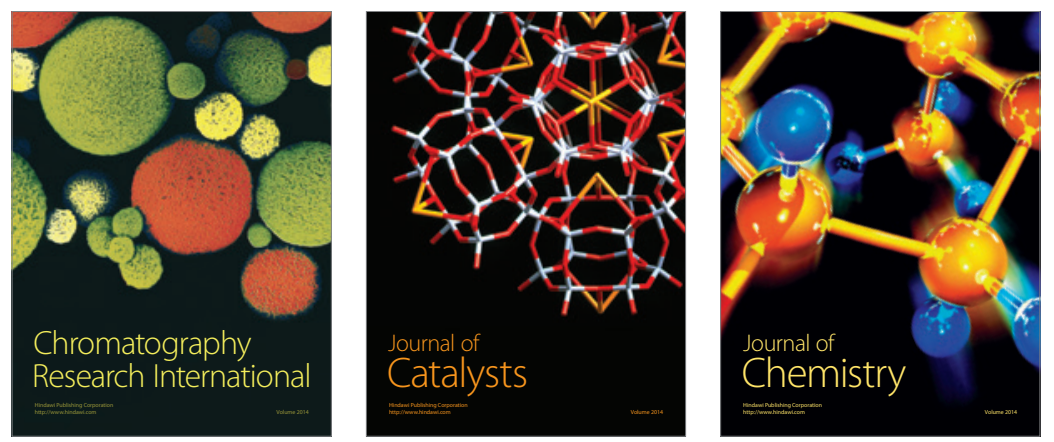
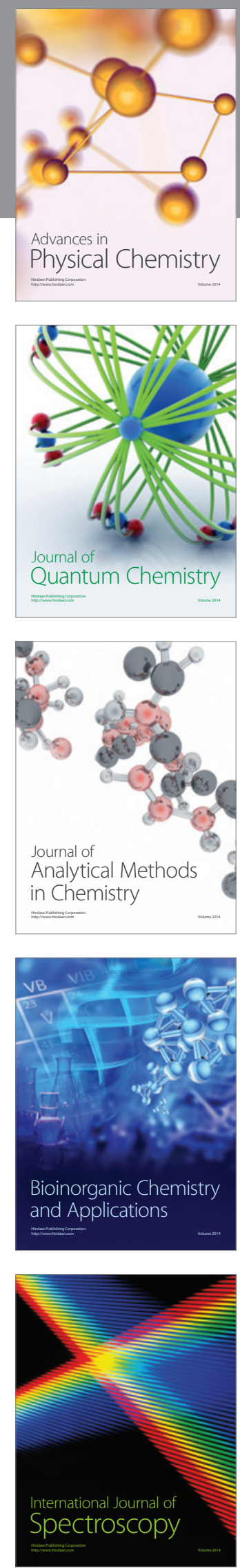